\title{
Sex Hormones Regulate Hepatic Fetuin Expression in Male and Female Rats
}

\author{
Sang Woo Kim ${ }^{a}$ Jung-Won Choi ${ }^{a}$ Dong Seok Lee ${ }^{b}$ Jong Won Yun ${ }^{a}$ \\ aDepartment of Biotechnology, Daegu University, Kyungsan, Kyungbuk, 'bSchool of Life Science \\ and Biotechnology, Kyungpook National University, Daegu, Republic of Korea
}

\section{Key Words}

Dihydrotestosterone $\cdot 17 \beta$-estradiol $\bullet$ Fetuin $\cdot$ Liver $\cdot$ Sex hormones

\begin{abstract}
Background: To date, there are limited studies on the sex-specific relationship between fetuins (Ft-A and Ft-B) and metabolic diseases. Our recent proteomic study has shown that fetuins may play sex-dependent roles in obesity and diabetes. In the present study, we investigated the expression of hepatic fetuins with respect to the effects of sex hormones both in vivo and in vitro. Methods \& Results: A sex hormone-treated rat model was established in order to study the effects of sex hormones on hepatic fetuin expression. Animal experiments revealed that $17 \beta$-estradiol (E2)- and dihydrotestosterone (DHT)-treated rats showed opposite effects in terms of body weight gain in both genders. Interestingly, Ft-A and Ft-B were sex-dependently expressed in the livers of rats, responding to different regulatory modes of sex hormone receptors $(E R \alpha, E R \beta$, and $A R)$. To validate in vivo data, rat normal liver cells were treated with E2 or DHT at different concentrations, and similar expression patterns as those in the animalbased experiments were confirmed. We found that these changes were mediated via sex hormone receptors using antagonist experiments. Conclusion: The results of the present study indicate that sex hormones induce gender-dimorphic expression of hepatic fetuins directly via sex hormone receptors. To the best of our knowledge, this is the first approach to address the effects of sex hormones on hepatic fetuin expression.
\end{abstract}

Copyright (C) 2014 S. Karger AG, Basel

S.W. Kim and J.-W. Choi contributed equally to this study.

Dr. Jong Won Yun

Department of Biotechnology, Daegu University, Kyungsan

Kyungbuk 712-714, (Republic of Korea)

Tel. +82-53-850-6556, Fax +82-53-850-6559, E-Mail jwyun@daegu.ac.kr 
Kim/Choi/Lee/Yun: Sex Hormones Regulate Hepatic Fetuin Expression

\section{Introduction}

Steroid biosynthesis and metabolism are essential in controlling physiological functionality in various species, and defects in these processes are related to many multifactorial diseases, including cancer, diabetes, and neurological diseases [1]. Estrogen is a steroid compound and plays a role as the primary female sex hormone [2]. It is a major regulator of energy balance, body weight, fat distribution, and appetite in mice [3], and ovariectomized mice show increased food consumption and fat mass, which can be reversed by estrogen replacement [4]. In addition, estrogen suppresses lipogenesis and triglyceride (TG) accumulation in the adipose tissue and livers of high-fat diet (HFD)-fed as well as leptin-deficient female mice [5]. Moreover, a rat vascular injury model demonstrated an early anti-inflammatory effect of estrogen in a sexually-dimorphic manner [6]. Estrogen exerts its physiological effects through two estrogen receptor (ER) subtypes, ER $\alpha$ and ER $\beta$, which belong to the nuclear receptor family of ligand-activated transcription factors [7].

Testosterone is a main active androgen and can be either converted to estrogen in a reaction catalyzed by aromatase or transformed into another active form like dihydrotestosterone [8]. It plays a key role in carbohydrate, fat, and protein metabolism and affects body fat composition and muscle mass in males [9]. Thus, its deficiency is related to increased fat mass, TG, and cholesterol levels as well as decreased insulin sensitivity and impaired glucose tolerance $[9,10]$. Further, testosterone mediates its physiological effects through the activation of androgen receptor (AR), a ligand-activated transcription factor [11].

Estrogen is produced by the aromatization of androstenedione to estrone in ovaries, followed by its conversion to $17 \beta$-estradiol (E2), which acts on distant target tissues by circulation. However, in postmenopausal women and men, it is produced in extragonadal sites such as adipose tissue, vessels, and bone by aromatization from circulating testosterone $[11,12]$. Accordingly, testosterone should be considered as a circulating prohormone that is locally converted to either E2 or dihydrotestosterone (DHT), the main ligand of AR, in both males and females. Although DHT cannot be aromatized to estrogen directly, it can be converted to $5 \alpha$-androstane- $3 \beta$ or $17 \beta$-diol, which acts on ERs $[11,13]$.

Fetuins are abundant proteins in the blood that are expressed in hepatocytes and monocyte/macrophages [14]. Recently, Dietzel et al. [15] reported that Ft-B deficiency leads to premature hardening of the zona pellucida (ZP), a glycoprotein matrix surrounding mammalian oocytes, thereby inducing female infertility. To date, there is very limited information on fetuin-B (Ft-B), even though there are various studies on fetuin-A (Ft-A), the first member of the fetuin family [16]. Ft-A and Ft-B have similarities and differences in gene regulation as well as partially overlapping functions [16]. Earlier reports demonstrated that up-regulation of Ft-A results in insulin resistance [17] and obesity [18], as well as fat accumulation in the liver [19]. However, there are also contradictory results [20, 21]. In addition, Ft-A is divergently regulated by various proinflammatory mediators, and functions as a positive or negative acute phase protein in injury and infection [22]. On the other hand, it was found that Ft-B levels decreased during the acute phase of inflammation in rat models [16]. Recently, the role of Ft-A in mineralized matrix metabolism was presented that it is a mineral carrier protein and a systemic inhibitor of pathological mineralization complementing local inhibitors and acts in a cell- or tissue-restricted fashion [23]. We previously observed decreased plasma protein levels of both Ft-A and Ft-B in HFD-fed obese rats [24] as well as a possible association between reduced Ft-B levels and higher risk of diet-induced obesity through impaired fatty acid metabolism in hepatocytes for the first time [25].

To date, there are limited studies on the sex-specific association of fetuins with metabolic diseases. Laughlin et al. [26] reported that higher Ft-A concentrations in the blood are related to an increased risk of developing type 2 diabetes in older women, whereas they are not associated with diabetes risk in older men. In our earlier study, we found that plasma levels of both Ft-A and Ft-B are significantly lower in males after HFD feeding while 
Kim/Choi/Lee/Yun: Sex Hormones Regulate Hepatic Fetuin Expression

remarkably higher in females [27]. Moreover, Ft-B has been shown to be up-regulated in plasma of both male and female diabetic rats [28]. Those results led us to hypothesize that gender-dimorphic regulation of fetuins is associated with hormonal effects. Therefore, in the current study, we examined whether or not fetuins are differentially regulated by sex hormones, estrogen and testosterone, both in vivo and in vitro.

\section{Materials and Methods}

\section{Animal experiments}

Five-week-old male and female SLC Sprague-Dawley (SD) rats were purchased from Daehan Experiment Animals (Seoul, Korea) and acclimatized in a temperature $\left(23 \pm 2^{\circ} \mathrm{C}\right)$ and humidity $(55 \%)$ controlled room under a $12 \mathrm{hrs}$ day/night cycle for 1 week. After adaptation, animals were subdivided into six groups: male control ( $n=6)$, male E2 $(n=6)$, male DHT ( $n=6)$, female control $(n=6)$, female E2 $(n=6)$, and female DHT $(n=6)$. To determine the effects of sex hormones, both E2 and DHT were dissolved in 100\% alcohol, diluted in 0.15 $\mathrm{M} \mathrm{NaCl}$ solution, and then injected intraperitoneally at a final concentration of $750 \mu \mathrm{g}$ of sex hormone $/ \mathrm{kg}$ of body weight for 48 days at 3 day intervals. As a control, vehicle was injected at the same volume. All rats and food were weighed every week for 6 weeks. Both before and after the experiment, plasma levels of E2 and DHT were determined using a rat E2 (Cayman Chemical Company, Ann Arbor, MI, USA) and DHT ELISA kit (Bioassay Technology Laboratory, Shanghai, China). Rats were sacrificed after fasting for at least $12 \mathrm{hrs}$ on day 48 , after which the livers were isolated, washed in ice cold saline, and then stored at $-80^{\circ} \mathrm{C}$. Blood was rapidly withdrawn from the ends of rat tails into EDTA tubes (BD, Franklin Lakes, NJ, USA) under anesthesia by $3 \%$ diethyl ether. Blood samples were centrifuged (3000 $\times g, 10 \mathrm{~min})$, after which plasma was collected, frozen in liquid nitrogen, and stored at $-80^{\circ} \mathrm{C}$. The Committee for Laboratory Animal Use and Care of Daegu University approved all animal experiments. All trials were carried out in accordance with the Guide for the Care and Use of Laboratory Animals published by the National Institutes of Health.

\section{Preparation and stimulation of cells}

Clone 9 cells (C9; originated from livers of normal 4-week-old SD male rats) were purchased from the American Type Culture Collection (ATCC, Manassas, VA, USA) and cultured in Kaighn's Modified F-12 medium (ATCC) containing $2 \mathrm{mM} \mathrm{L-glutamine}$ and $1500 \mathrm{mg} / \mathrm{L}$ of sodium bicarbonate supplemented with $10 \%$ fetal bovine serum (FBS; Hyclone, Logan, UT, USA) in a $37^{\circ} \mathrm{C}$-humidified $5 \% \mathrm{CO}_{2}$ incubator. All reagents except for growth medium and FBS for cell culture were purchased from Invitrogen (Grand island, NY, USA). For stimulation of cells by the sex hormones E2 (Sigma-Aldrich, St. Louis, MO, USA) and DHT (SigmaAldrich), cells were plated in 6-well tissue culture plates (Costar, NY, USA) at a density of $5 \times 10^{5}$ cells/well 24 hrs prior to stimulation. Cells were harvested at $12 \mathrm{hrs}$ after treatment with sex hormones $(0,0.1,1$, or 10 $\mu \mathrm{M}$ ), followed by treatment with RIPA buffer (Sigma-Aldrich) for Western blot analysis and lysis buffer using a total RNA extraction kit (iNtRON Biotechnology, Seongnam, Korea) for quantitative real-time RT-PCR. To confirm changes in Ft-A and Ft-B expression via sex hormone receptors, ER antagonist (fulvestrant, SigmaAldrich) or AR antagonist (flutamide, Sigma-Aldrich) were used, respectively.

\section{Quantitative real-time RT-PCR}

Transcript levels of genes were quantitatively determined by real-time RT-PCR (Stratagene 246 mx 3000p QPCR System). Total RNA was isolated from cells using an easy-spinTM (DNA-free) Total RNA Extraction kit, and reverse-transcription was carried out using a Maxime RT Premix kit (iNtRON Biotechnology). We employed the SYBR Green Dye system (Applied Biosystems, Foster City, CA, USA) for real-time PCR. Transcript levels of each gene were normalized to GAPDH transcript levels. Sequences of primer sets are listed in Table 1.

\section{Western blot analysis}

Cell lysates were prepared with RIPA buffer, homogenized, and centrifuged at 12,000 $\times g$ for $20 \mathrm{~min}$. Cell extract was diluted in sample buffer ( $50 \mathrm{mM}$ Tris of pH 6.8, 2\% SDS, $10 \%$ glycerol, $0.1 \%$ bromophenol blue, and $5 \% \beta$-mercaptoethanol) and heated for $5 \mathrm{~min}$ before SDS-polyacrylamide gel electrophoresis (PAGE) at $6,8,10$, or $12 \%$. After electrophoresis, proteins were transferred to a polyvinylidene difluoride (PVDF; 
Kim/Choi/Lee/Yun: Sex Hormones Regulate Hepatic Fetuin Expression

Table 1. Sequences of primers used for real-time RT-PCRs

\begin{tabular}{lll}
\hline Genes & & Primer sequence $\left(5^{\prime}-3^{\prime}\right)$ \\
\hline \multirow{2}{*}{ Ahsg } & $\mathrm{F}^{a}$ & ACGTGGTCCACACTGTCAAA \\
& $\mathrm{R}^{b}$ & CGCAGCTATCACAAACTCCA \\
Fetub & $\mathrm{F}$ & CCCTGAGCAGGAGGAGCC \\
& $\mathrm{R}$ & CATCAGGGGGTTCTTTGCTTT \\
Esr1 & $\mathrm{F}$ & CATCGATAAGAACCGGAGGA \\
& $\mathrm{R}$ & AAGGTTGGCAGCTCTCATGT \\
Esr2 & $\mathrm{F}$ & AAAGTAGCCGGAAGCTGACA \\
& $\mathrm{R}$ & CTCCAGCAGCAGGTCATACA \\
Ar & $\mathrm{F}$ & CCCTGTGTGTGCAGCTAGAA \\
\multirow{2}{*}{ Internal control } & \\
\multirow{2}{*}{ Gapdh } & $\mathrm{R}$ & AGACAGGATCTGCCCTGCTA \\
& $\mathrm{F}$ & AGACAGCCGCATCTTCTTGT \\
\hline
\end{tabular}

$\overline{{ }^{a} \mathrm{~F} \text {, sequence from sense strands; }{ }^{b} \mathrm{R} \text {, sequence from anti-sense strands }}$

Santa Cruz Biotechnology, Santa Cruz, CA, USA) membrane and blocked for $1 \mathrm{hr}$ with 5\% skim milk in TBS (Tris- buffered saline)-T buffer (10 mM Tris-HCl, $150 \mathrm{mM} \mathrm{NaCl}, 0.1 \%$ Tween 20 containing). The membrane was then rinsed with three changes of TBS-T buffer, followed by incubation for $1 \mathrm{hr}$ with a 1:1000 dilution of primary polyclonal antibody (anti- $\beta$-actin, Ft-A, Ft-B, ER $\alpha$, ER $\beta$ and AR; Santa Cruz Biotechnology) in TBS-T buffer containing 1\% skim milk. After three washes, the membrane was incubated for $1 \mathrm{hr}$ with horseradish peroxidase-conjugated anti-mouse IgG, anti-goat IgG, or anti-rabbit IgG (1:1000, Santa Cruz Biotechnology) in TBS-T buffer containing 1\% skim milk and developed by enhanced chemiluminescence (ECL; iNtRON Biotechnology). Western blot analysis was recorded using medical X-ray film (AGPA, CP-BU new, Mortsel, Belgium), scanned using a UMAX PowerLook 1120 (Maximum Technologies, Akron, OH, USA), and digitalized using image analysis software (KODAK 1D, Eastman Kodak, Rochester, NY, USA).

\section{Enzyme-linked immunosorbent assay (ELISA)}

Secreted Ft-A and Ft-B levels were measured using ELISA. A commercial kit purchased from Aviscera Bioscience (Santa Clara, CA, USA) was used for determination of Ft-A. In addition, a CUSABIO Life Science ELISA kit (Wuhan, China) was used for measurement of Ft-B levels. Plasma biochemical characterizations of rats were carried out using a commercial ELISA kit to measure levels of plasma total cholesterol (TC), and triglyceride (TG; Biovision Inc. Milpitas, CA, USA). Each assay was performed in triplicate using C9 cell culture supernatant or individual plasma samples of six selected rats per group. Measurement and analysis were performed according to the manufacturer's instructions.

\section{Statistical analysis}

Statistical significance between the control and hormone-treated groups were compared using oneway ANOVA. Group means were considered significantly different at $p<0.05$. The significance of the effects of sex hormonal treatments was determined using multivariate ANOVA (M-ANOVA). All statistical tests were performed using SPSS 17.0 software (Technologies, Chicago, IL, USA) at a significance level of $p<0.05$.

\section{Results}

Variances in physiological parameters of sex hormone-treated rats

We established a sex hormone-treated animal model in males and females in order to investigate the effects of sex hormones on hepatic fetuin expression. After treatment with sex hormones (E2 or DHT) in SD rats, E2- and DHT-treated rats showed opposite effects in terms of body weight gain in both genders (Fig. 1A). Body weight gain was significantly suppressed by E2 treatment in both males and females, whereas DHT showed distinct stimulatory effects on body weight gain in females only (Fig. 1A). E2 treatment led to lower food efficiency (total body weight gain per total food intake) in both genders, which was in agreement with the changes in body weight gain between control and sex hormone-treated groups (Fig. 1B). We 
Fig. 1. Body weight (A), food efficiency (B), and plasma levels of total cholesterol (TC) (C), triglyceride (TG) (D), $17 \beta$-estradiol (E2) (E), and dihydrotestosterone (DHT) $(\mathrm{F})$ in control (CON), E2-treated (E2), and DHT-treated (DHT) male and female rats. Statistical significance between each group was calculated by ANOVA, where ${ }^{*} p<0.05$ and ${ }^{* *} p<0.01$. Significance of the effects of sex hormonal treatments was tested using M-ANOVA, where ${ }^{\dagger} p<0.05$ and ${ }^{\dagger} p<0.01$.
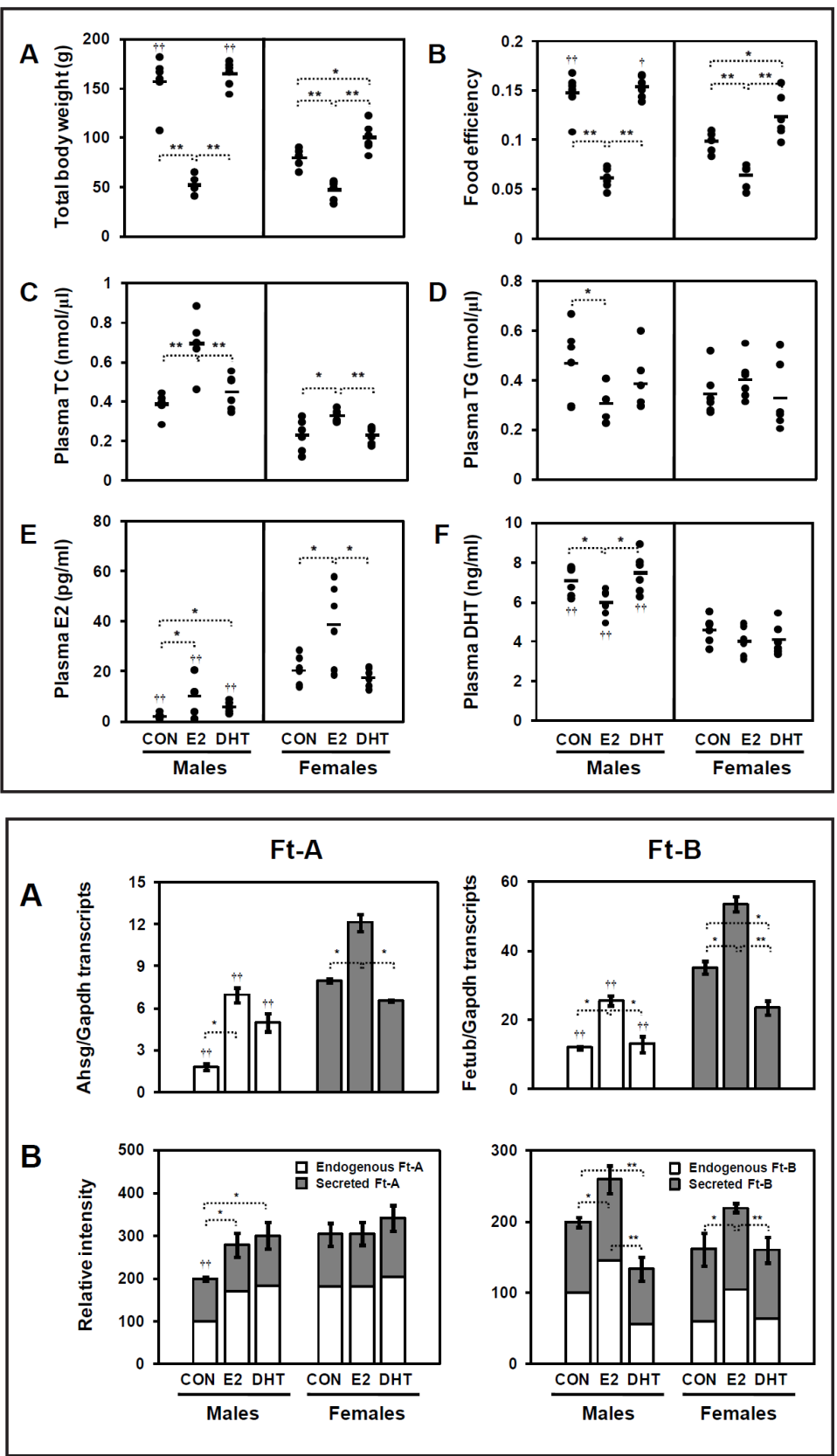

Fig. 2. Expression of Ft-A and Ft-B in livers of rats after sex hormonal treatment. mRNA (A) as well as endogenous and secreted protein levels (B). Relative intensity means sum of endogenous protein (white bars) and secreted protein (gray bars) levels and was determined as follows: relative intensity $=\mathrm{a}+\mathrm{b} \quad(\mathrm{a}$, divided endogenous protein levels of each group by those of control group and multiplied values by $100 ; \mathrm{b}$, divided secreted protein levels of each group by those of control group and multiplied values by 100). Statistical significance between each group was determined by ANOVA, where ${ }^{*} p<0.05$ and ${ }^{* *} p<0.01$. Significance of the effects of sex hormonal treatment was tested using M-ANOVA, where ${ }^{\dagger \dagger} p<0.01$.

found that plasma biochemical parameters reflected elevation of TC and attenuation of TG in the E2-treated group (Fig. 1C and 1D). In addition, plasma E2 concentration was significantly higher in the E2-treated group in both genders, whereas plasma DHT levels were remarkably lower in E2-treated male rats only (Fig. 1E and 1F).

Changes in hepatic fetuin- $A$ and fetuin-B expression by sex hormonal treatment

We investigated the expression levels of Ft- $\mathrm{A}$ and $\mathrm{Ft}-\mathrm{B}$ in sex hormone-treated rat livers of males and females. It was found that expression of both the Ft-A- and Ft-B-encoding genes (Ahsg and Fetub) were elevated by E2 in both males and females, whereas their expression levels were reduced or unchanged by DHT treatment (Fig. 2A). Although protein levels of 


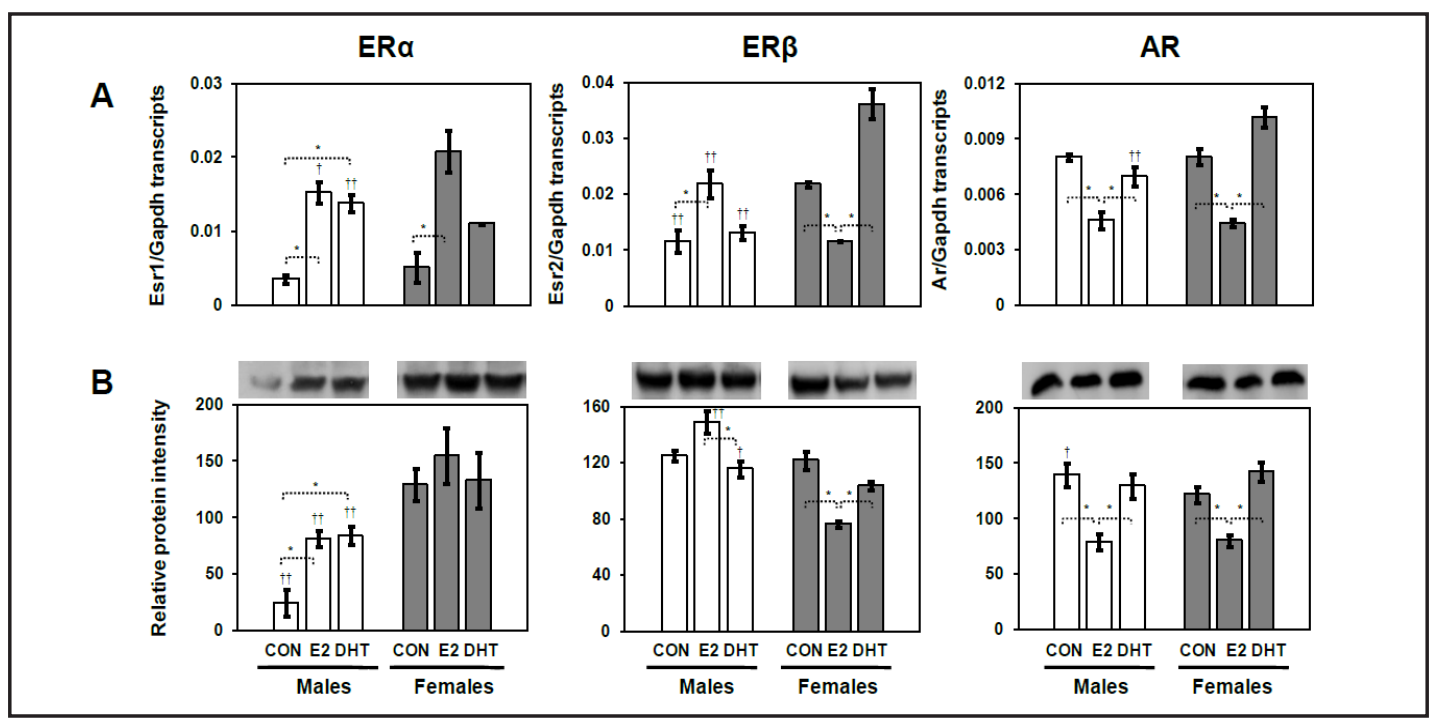

Fig. 3. Expression of sex hormone receptors in the livers of rats after sex hormonal treatment. mRNA (A) and endogenous protein levels (B). Statistical significance between each group was determined by ANOVA, where ${ }^{*} p<0.05$. Significance of the effects of sex hormonal treatments was tested using M-ANOVA, where ${ }^{\dagger} p<0.05$ and ${ }^{\dagger \dagger} p<0.01$.

Fig. 4. Effects of sex hormonal treatment (A) and sex hormones as well as their receptor antagonists (B and C) on Ft-A and Ft-B expression in normal rat liver cells (C9). Relative intensity means sum of endogenous protein (white bars) and secreted protein (gray bars) levels and was determined as follows: relative intensity $=\mathrm{a}+\mathrm{b}(\mathrm{a}$, divided endogenous protein levels of each group by those of control group and multiplied values by $100 ;$ b, divided secreted protein levels of each group by those of control group and multiplied values by 100). Statistical significance between controls, sex hormonetreated groups, and antagonist-treated groups was determined by ANOVA, where ${ }^{*} p<0.05$ and ${ }^{* *} p<0.01$.
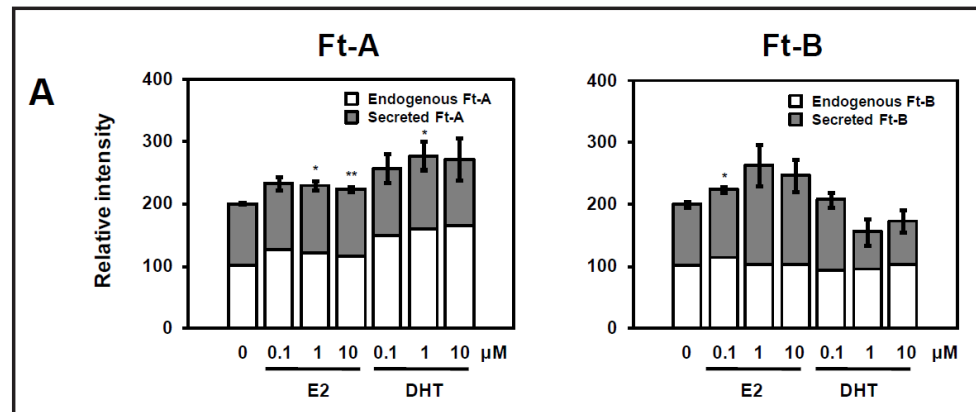

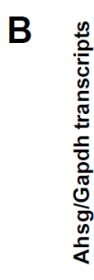
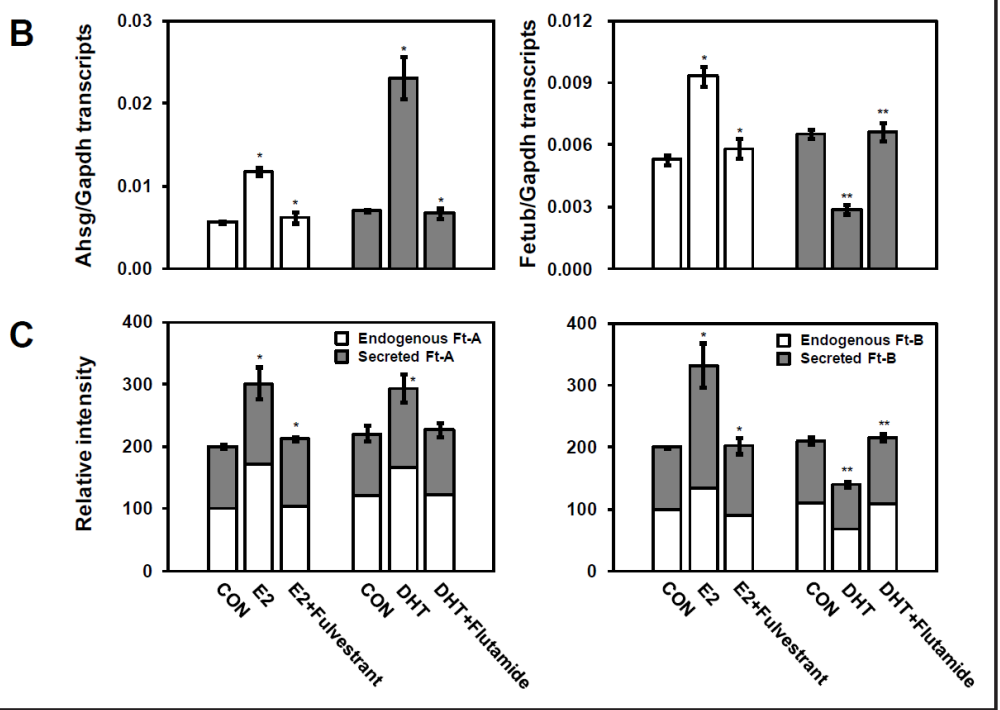

Ft-B (sum of endogeneous and secreted Ft-B) were in line with transcript levels, Ft-A protein levels in response to sex hormonal treatment were not in agreement with transcript levels (Fig. 2B). 


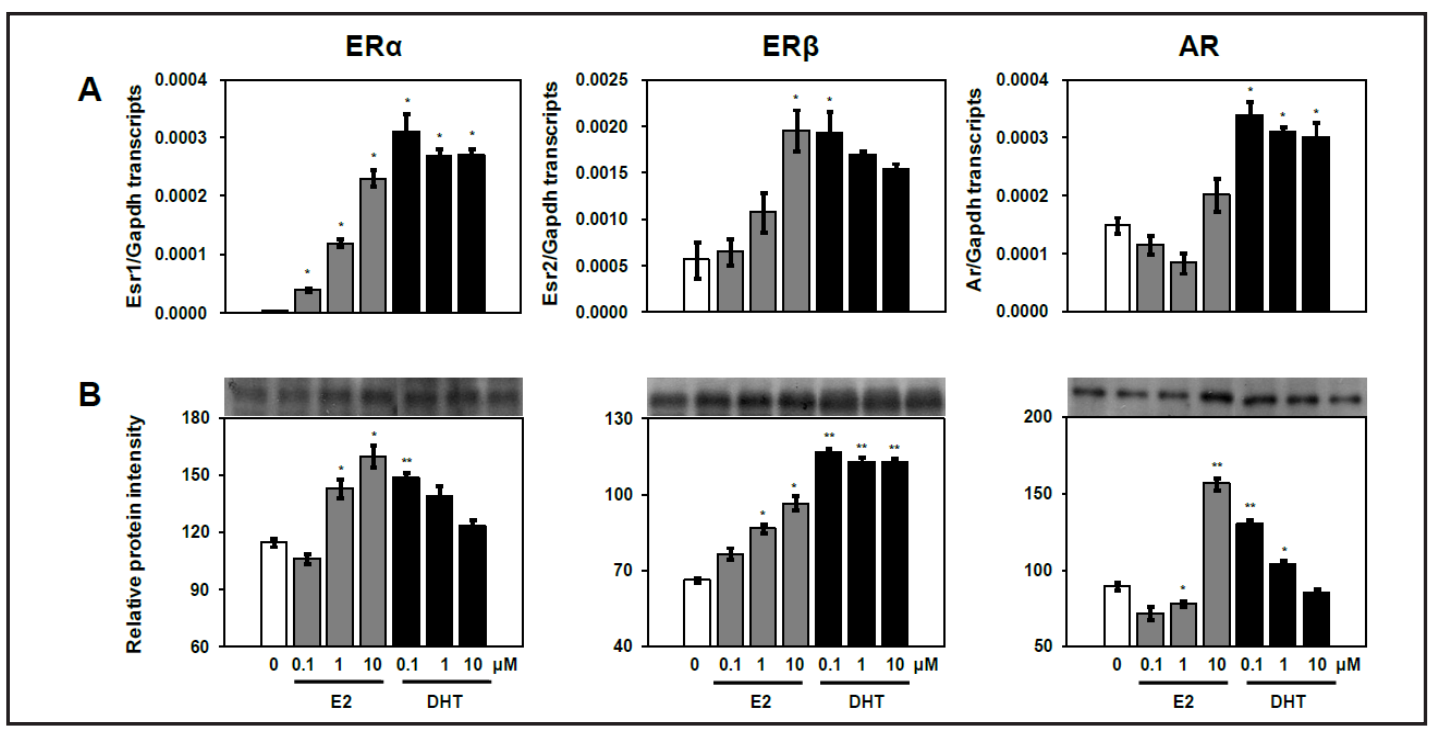

Fig. 5. Effects of sex hormonal treatment on mRNA (A) and endogenous protein (B) levels of sex hormone receptors in normal rat liver cells (C9). Statistical significance between controls and sex hormone-treated groups was determined by ANOVA test, where ${ }^{*} p<0.05$ and ${ }^{* *} p<0.01$.

Changes in hepatic sex hormone receptors by sex hormonal treatment

We also investigated the expression levels of sex hormone receptors (e.g. Er $\alpha, \operatorname{Er} \beta$, and AR) in sex hormone-treated rat livers of males and females. Both transcript (Fig. 3A) and protein (Fig. 3B) expression of these receptors were significantly gender-dependent, indicating a positive regulatory effect of E2 on both ER $\alpha$ and ER $\beta$ as well as a negative regulatory effect on AR. It is noteworthy that $\mathrm{E} 2$ reduced both transcript and protein levels of ER $\beta$ only in females (Fig. 3).

\section{Inhibitory effects of antagonists on sex hormone receptors}

To further investigate the regulation of Ft-A and Ft-B by sex hormone receptors, rat normal liver cells (clone 9, C9) were treated with E2 or DHT at different concentrations $(0,0.1,1$, or $10 \mu \mathrm{M})$ for $12 \mathrm{hrs}$, after which protein levels of endogenous and secreted Ft-A and Ft-B were determined by Western blot analysis and ELISA, respectively. The levels of both endogenous and secreted fetuin proteins were determined as a total sum. Although sex hormonal treatment did not have a dramatic dose-dependent effect on Ft-A or Ft-B expression, Ft-A was elevated by both E2 and DHT, whereas Ft-B was up-regulated by E2 and down-regulated by DHT at all concentrations (Fig. 4A). This result suggests that fetuins showed similar expression patterns as the animal-based results. Next, we investigated whether or not changes in expression of Ft-A and Ft-B by sex hormones are induced via sex hormone receptors using sex hormone receptor antagonists in C9 cells. C9 cells were treated with sex hormones and sex hormone receptor antagonists, after which the cells and cell culture supernatant were harvested after $12 \mathrm{hrs}$ in order to determine mRNA, endogenous protein, and secreted protein levels of Ft-A and Ft-B. It was found that addition of either the ER antagonist fulvestrant $(0.1 \mu \mathrm{M})$ or AR antagonist flutamide $(0.1 \mu \mathrm{M})$ to C9 cells completely abolished E2- or DHT-induced up- or down-regulation of both Ft-A and Ft-B (Fig. 4B and 4C), which suggests that regulation of Ft-A and Ft-B expression by E2 or DHT was obviously mediated via activation of ER or AR. In the absence of E2 or DHT, mRNA, endogenous protein, and secreted protein levels of Ft-A and Ft-B showed no significant change (data not shown).

Changes in expression of sex hormone receptors by sex hormonal treatment

We investigated changes in the expression of sex hormone receptors as well as fetuins in E2- or DHT-treated C9 cells. As shown in Fig. 5, transcript and endogenous protein levels 
of ER $\alpha$ and ER $\beta$ were up-regulated by E2 or DHT treatment at certain concentrations, but the effects were not dose-dependent (Fig. 5). In contrast, expression of AR was very sensitive to the concentrations of sex hormones, indicating that $\mathrm{E} 2$ was a positive regulator of $\mathrm{AR}$ at high concentrations $(10 \mu \mathrm{M})$ while a negative regulator at lower concentrations $(0.1 \sim 1 \mu \mathrm{M}$, Fig. $5)$.

\section{Discussion}

The most remarkable outcome of this study is the involvement of sex hormones in the regulation of fetuins. Specifically, we found that both Ft-A and Ft-B were significantly increased by E2, whereas Ft-A was remarkably increased and Ft-B significantly reduced by DHT. However, there were negligible differences in expression among mRNA, endogenous protein, and secreted protein levels.

We established a sex hormone-treated rat model to investigate the effects of sex hormones on hepatic fetuin expression. Sex hormones and their receptors are well-known regulators of lipid and carbohydrate metabolism, and their impaired signaling is related to the development of metabolic disease [7, 29, 30]. Especially, estrogen reduces body weight gain in HFD-fed mice [5], decreases hepatic lipogenensis, and improves insulin sensitivity [31]. Therefore, estrogen may have anti-obesity effects in rodents. Meanwhile, testosterone is an important regulator of fat mass and distribution in males $[9,10]$. In females, however, DHT induces a myogenic transcriptional program in intra-abdominal adipose tissue [32] as well as formation of typical polycystic ovaries with increased apoptotic follicles in rats [33]. Specifically, DHT-treated rats develop obesity with enlarged adipocyte size and insulin resistance, indicating that increased androgen levels may induce alteration of body composition as well as reduction of insulin sensitivity [33]. In our study, E2 treatment also led to striking reduction of food efficiency in both genders, whereas DHT treatment induced significant stimulation of food efficiency in females only (Fig. 1B). In addition, increased plasma TC and decreased plasma TG were observed in E2-treated group (Fig. 1C and 1D). E2 treatment led to decreased body weight and plasma TG content. Our finding is similar to the studies conducted by Bryzgalova et al. [5] who demonstrated that E2 treatment in HFD-fed mice resulted in decrease body weight, plasma leptn levels, and hepatic TG content. Therefore, reduction in TG content in the liver upon E2 treatment may contribute to the improvement of insulin sensitivity. Notably, Ft-A and Ft-B were remarkably up-regulated in E2-treated cells and livers of E2-treated male rats (Figs. 2 and 4). These results are in line with our earlier finding that Ft-A and Ft-B levels are significantly reduced in HFD-fed male rats [27]. Indeed, E2 induced similar changes in Ft-A and Ft-B expression, whereas DHT induced the opposite expression patterns (Figs. 2 and 4). It could be inferred that these changes are due to functional differences between Ft-A and Ft-B possibly through the testosterone-AR signaling pathway.

We confirmed that changes in Ft-A and Ft-B expression by sex hormones are induced via sex hormone receptors by treating sex hormone receptor antagonists to C9 cells. Estrogen signaling arises due to the direct binding of dimerized ER to estrogen-responsive elements (ERREs) in the regulatory regions of estrogen target genes. The cellular response to androgens is mediated via AR and ligand-inducible transcription factor [34]. After ligand binding, cytosolic AR undergoes conformational changes and interacts with coregulatory molecules, which facilitate nuclear translocation and dimerization [35]. In this study, we found that E2- or DHT-induced up- or down-regulation of Ft-A and Ft-B was completely abolished by addition of ER antagonist (fulvestrant) or AR antagonist (flutamide) in rat liver cells (Fig. 4B and 4C). These data suggest that regulation of Ft-A and Ft-B expression by E2 or DHT is mediated via activation of ER or AR. In particular, E2 acted as a positive and negative regulator of ER and AR, respectively, thereby influencing sex-dimorphic regulation of fetuins.

Here, we investigated changes in the expression of sex hormone receptors as well as fetuins by sex hormonal treatment. Estrogen treatment has been shown to increase both 
ER and ER mRNA levels in the livers of male rats [36-38]. In comparison, estrogen has been shown to induce down-regulation of AR in cultured human mammary cancer cells [39]. These results are in line with our finding that ER $\alpha$ and ER $\beta$ were significantly up-regulated in both E2-treated cells as well as livers of male rats, whereas AR was remarkably altered depending on the E2 concentration and actually reduced in E2-treated males (Figs. 3 and 5).

Hepatic AR is an inducible protein whose synthesis is regulated by its own ligands, which include androgens acting as positive regulators and estradiol as negative ones [40]. Testosterone can bind to both ER and AR and thus influence mechanisms of action related to sex hormones, as it can be converted to E2 by aromatase [11]. In the current study, we found that $E R \alpha, E R \beta$, and AR were significantly up-regulated in DHT-treated cells, whereas only ER $\alpha$ was remarkably elevated in DHT-treated male rats (Figs. 3 and 5). Taken together, these data suggest that expression of sex hormone receptors is influenced by sex hormones, but the effects vary according to supplemental factors such as hormone concentrations, age, species, and environmental factors.

In the present study, transcript and protein levels of sex hormone receptors as well as fetuins induced by sex hormones were not significantly different. Exact prediction of protein levels from mRNA transcript levels is impossible and current techniques for quantitative analysis of mRNA transcript and protein expression levels have limitations [41]. The correlation between transcript and protein levels can be as little as $40 \%$ depending on the system [42]. There are numerous factors that affect this correlation, including the various transcription and translation processes, protein stability, half-life of different proteins, degradation rate of mRNA, lower rate of mRNA transcription compared to protein translation, and protein diversity [42]. Although transcriptional data are useful for studying protein levels, gene expression is frequently not reflected at the protein level [42]. Moreover, there were some differences between in vivo and in vitro experiments. In vitro models are widely used in experimental biology, and thus it is reasonable to ask how effective these models reflect an actual animal [43]. In fact, no in vitro condition creates new pathways or biology, and no culture system can exactly mimic in vivo development, thereby creating contrary results between in vitro and in vivo data [44].

The current data demonstrate that E2 and DHT alter the expression of hepatic fetuins through mechanisms involving the sex hormone receptors ER $\alpha, E R \beta$, and AR. These results were confirmed using both an animal model and sex hormone-treated cells. Especially, we found different responses of Ft-A and Ft-B expression to DHT. These results could offer new solutions for explaining functional differences in Ft-A and Ft-B expression in the testosteroneAR signaling pathway. Further studies are required to directly address the physiological role of fetuins in sex hormone related-signaling pathways. Nonetheless, to the best of our knowledge, this is the first approach to address the effects of sex hormones on hepatic fetuin expression.

\section{Acknowledgements}

This work was supported by the Mid-career Researcher Program (2013R1A2A2A05004195) and the SRC Program (Center for Food \& Nutritional Genomics, grant number 2008-0062618) through an NRF grant funded by the Ministry of Science, ICT and Future Planning, Korea.

\section{Disclosure Statement}

The authors have declared no conflict of interest. 
Kim/Choi/Lee/Yun: Sex Hormones Regulate Hepatic Fetuin Expression

\section{References}

1 Valsamakis G, Anwar A, Tomlinson JW, Shackleton CH, McTernan PG, Chetty R, Wood PJ, Banerjee AK, Holder G, Barnett AH, Stewart PM, Kumar S: 11beta-hydroxysteroid dehydrogenase type 1 activity in lean and obese males with type 2 diabetes mellitus. J Clin Endocrinol Metab 2004;89:4755-4761.

2 Wei J, Shi Y, Zhang X, Feng Y, Luo G, Zhang J, Mu Q, Tang Y, Yu Y, Pan L, Nilsson-Ehle P, Xu N: Estrogen upregulates hepatic apolipoprotein m expression via the estrogen receptor. Biochim Biophys Acta 2011;1811:1146-1151.

3 Dubuc PU: Effects of estrogen on food intake, body weight, and temperature of male and female obese mice. Proc Soc Exp Biol Med 1985;180:468-473.

4 Laudenslager ML, Wilkinson CW, Carlisle HJ, Hammel HT: Energy balance in ovariectomized rats with and without estrogen replacement. Am J Physiol 1980;238:R400-405.

-5 Bryzgalova G, Lundholm L, Portwood N, Gustafsson JA, Khan A, Efendic S, Dahlman-Wright K: Mechanisms of antidiabetogenic and body weight-lowering effects of estrogen in high-fat diet-fed mice. Am J Physiol Endocrinol Metab 2008;295:E904-912.

6 Chen SJ, Li H, Durand J, Oparil S, Chen YF: Estrogen reduces myointimal proliferation after balloon injury of rat carotid artery. Circulation 1996;93:577-584.

7 Faulds MH, Zhao C, Dahlman-Wright K, Gustafsson JA: The diversity of sex steroid action: Regulation of metabolism by estrogen signaling. J Endocrinol 2012;212:3-12.

8 Simard J, Ricketts ML, Gingras S, Soucy P, Feltus FA, Melner MH: Molecular biology of the 3beta-hydroxysteroid dehydrogenase/delta5-delta4 isomerase gene family. Endocr Rev 2005;26:525-582.

-9 Kelly DM, Jones TH: Testosterone: A metabolic hormone in health and disease. J Endocrinol 2013;217:R2545.

10 Jones TH: Testosterone deficiency: A risk factor for cardiovascular disease? Trends Endocrinol Metab 2010;21:496-503.

11 Mauvais-Jarvis F: Estrogen and androgen receptors: Regulators of fuel homeostasis and emerging targets for diabetes and obesity. Trends Endocrinol Metab 2011;22:24-33.

12 Simpson ER: Sources of estrogen and their importance. J Steroid Biochem Mol Biol 2003;86:225-230.

13 Weihua Z, Lathe R, Warner M, Gustafsson JA: An endocrine pathway in the prostate, erbeta, ar, 5alpha-androstane-3beta, 17beta-diol, and cyp7b1, regulates prostate growth. Proc Natl Acad Sci U S A 2002;99:13589-13594.

14 Dziegielewska K, Brown WM, Deal A, Foster KA, Fry EJ, Saunders NR: The expression of fetuin in the development and maturation of the hemopoietic and immune systems. Histochem Cell Biol 1996;106:319-330.

15 Dietzel E, Wessling J, Floehr J, Schafer C, Ensslen S, Denecke B, Rosing B, Neulen J, Veitinger T, Spehr M, Tropartz T, Tolba R, Renne T, Egert A, Schorle H, Gottenbusch Y, Hildebrand A, Yiallouros I, Stocker W, Weiskirchen R, Jahnen-Dechent W: Fetuin-b, a liver-derived plasma protein is essential for fertilization. Dev Cell 2013;25:106-112.

16 Olivier E, Soury E, Ruminy P, Husson A, Parmentier F, Daveau M, Salier JP: Fetuin-b, a second member of the fetuin family in mammals. Biochem J 2000;350 Pt 2:589-597.

17 Mathews ST, Singh GP, Ranalletta M, Cintron VJ, Qiang X, Goustin AS, Jen KL, Charron MJ, Jahnen-Dechent W, Grunberger G: Improved insulin sensitivity and resistance to weight gain in mice null for the ahsg gene. Diabetes 2002;51:2450-2458.

18 Reinehr T, Roth CL: Fetuin-a and its relation to metabolic syndrome and fatty liver disease in obese children before and after weight loss. J Clin Endocrinol Metab 2008;93:4479-4485.

19 Lin X, Braymer HD, Bray GA, York DA: Differential expression of insulin receptor tyrosine kinase inhibitor (fetuin) gene in a model of diet-induced obesity. Life Sci 1998;63:145-153.

20 Mori K, Emoto M, Yokoyama H, Araki T, Teramura M, Koyama H, Shoji T, Inaba M, Nishizawa Y: Association of serum fetuin-a with insulin resistance in type 2 diabetic and nondiabetic subjects. Diabetes Care 2006;29:468.

-21 Gunduz FO, Yildirmak ST, Temizel M, Faki Y, Cakmak M, Durmuscan M, Sezgin F: Serum visfatin and fetuin-a levels and glycemic control in patients with obese type 2 diabetes mellitus. Diabetes Metab J 2011;35:523528.

22 Wang H, Sama AE: Anti-inflammatory role of fetuin-a in injury and infection. Curr Mol Med 2012;12:625633. 
Kim/Choi/Lee/Yun: Sex Hormones Regulate Hepatic Fetuin Expression

23 Jahnen-Dechent W, Heiss A, Schafer C, Ketteler M: Fetuin-a regulation of calcified matrix metabolism. Circ Res 2011;108:1494-1509.

24 Choi JW, Wang X, Joo JI, Kim DH, Oh TS, Choi DK, Yun JW: Plasma proteome analysis in diet-induced obesity-prone and obesity-resistant rats. Proteomics 2010;10:4386-4400.

25 Choi JW, Liu H, Mukherjee R, Yun JW: Downregulation of fetuin-b and zinc-alpha2-glycoprotein is linked to impaired fatty acid metabolism in liver cells. Cell Physiol Biochem 2012;30:295-306.

-26 Laughlin GA, Barrett-Connor E, Cummins KM, Daniels LB, Wassel CL, Ix JH: Sex-specific association of fetuin-a with type 2 diabetes in older community-dwelling adults: The rancho bernardo study. Diabetes Care 2013;36:1994-2000.

-27 Liu H, Choi JW, Yun JW: Gender differences in rat plasma proteome in response to high-fat diet. Proteomics 2012;12:269-283.

28 Choi JW, Aseer KR, Chaudhari HN, Mukherjee R, Choi M, Yun JW: Gender dimorphism in regulation of plasma proteins in streptozotocin-induced diabetic rats. Proteomics 2013;13:2482-2494.

-29 Basu R, Dalla Man C, Campioni M, Basu A, Nair KS, Jensen MD, Khosla S, Klee G, Toffolo G, Cobelli C, Rizza RA: Effect of 2 years of testosterone replacement on insulin secretion, insulin action, glucose effectiveness, hepatic insulin clearance, and postprandial glucose turnover in elderly men. Diabetes Care 2007;30:19721978.

-30 Moverare-Skrtic S, Venken K, Andersson N, Lindberg MK, Svensson J, Swanson C, Vanderschueren D, Oscarsson J, Gustafsson JA, Ohlsson C: Dihydrotestosterone treatment results in obesity and altered lipid metabolism in orchidectomized mice. Obesity (Silver Spring) 2006;14:662-672.

-31 Gao H, Bryzgalova G, Hedman E, Khan A, Efendic S, Gustafsson JA, Dahlman-Wright K: Long-term administration of estradiol decreases expression of hepatic lipogenic genes and improves insulin sensitivity in ob/ ob mice: A possible mechanism is through direct regulation of signal transducer and activator of transcription 3. Mol Endocrinol 2006;20:1287-1299.

32 De Giorgio MR, Yoshioka M, St-Amand J: A single dose of dihydrotestosterone induced a myogenic transcriptional program in female intra-abdominal adipose tissue. J Steroid Biochem Mol Biol 2010;122:53-64.

-33 Manneras L, Cajander S, Holmang A, Seleskovic Z, Lystig T, Lonn M, Stener-Victorin E: A new rat model exhibiting both ovarian and metabolic characteristics of polycystic ovary syndrome. Endocrinology 2007;148:3781-3791.

-34 Sampson N, Neuwirt H, Puhr M, Klocker H, Eder IE: In vitro model systems to study androgen receptor signaling in prostate cancer. Endocr Relat Cancer 2013;20:R49-64.

-35 Cutress ML, Whitaker HC, Mills IG, Stewart M, Neal DE: Structural basis for the nuclear import of the human androgen receptor. J Cell Sci 2008;121:957-968.

-36 Koritnik DR, Koshy A, Hoversland RC: 17 beta-estradiol treatment increases the levels of estrogen receptor and its mrna in male rat liver. Steroids 1995;60:519-529.

37 Eisenfeld AJ, Aten RF: Estrogen receptors and androgen receptors in the mammalian liver. J Steroid Biochem 1987;27:1109-1118.

- 38 Stavreus-Evers A, Parini P, Freyschuss B, Elger W, Reddersen G, Sahlin L, Eriksson H: Estrogenic influence on the regulation of hepatic estrogen receptor-alpha and serum level of angiotensinogen in female rats. J Steroid Biochem Mol Biol 2001;78:83-88.

39 Stover EP, Krishnan AV, Feldman D: Estrogen down-regulation of androgen receptors in cultured human mammary cancer cells (mcf-7). Endocrinology 1987;120:2597-2603.

40 Roy AK, Milin BS, McMinn DM: Androgen receptor in rat liver: Hormonal and developmental regulation of the cytoplasmic receptor and its correlation with the androgen-dependent synthesis of alpha2u-globulin. Biochim Biophys Acta 1974;354:213-232.

41 Gygi SP, Rochon Y, Franza BR, Aebersold R: Correlation between protein and mrna abundance in yeast. Mol Cell Biol 1999;19:1720-1730.

42 Vogel C, Marcotte EM: Insights into the regulation of protein abundance from proteomic and transcriptomic analyses. Nat Rev Genet 2012;13:227-232.

43 Novakofski J: Adipogenesis: Usefulness of in vitro and in vivo experimental models. J Anim Sci 2004;82:905-915.

44 Liu MG, Li H, Xu X, Barnstable CJ, Zhang SS: Comparison of gene expression during in vivo and in vitro postnatal retina development. J Ocul Biol Dis Infor 2008;1:59-72. 